\title{
Thin Layer Chromatography (TLC) and Phytochemical Analysis of Moringa Oleifera Methanol, Ethanol, Water and Ethyl Acetate Extracts
}

Ahlam Rashed ${ }^{2 *}$, Ameerah Shaeroun ${ }^{2}$, Ahmed Belgasem Ahmed ${ }^{2}$, Hamed Alqamoudy ${ }^{2}$, Khalifa.S.Mohamed ${ }^{4}$, Nadea Almunir $^{2}$, Nouri Kushlaf ${ }^{2}$, A.M.EL-mahmoudy ${ }^{2}$, Akram Almabrouk misbah ${ }^{3}$, Seham TM Oshkondali ${ }^{1}$, and Zuhur rajab Almesai $^{2}$

${ }^{1}$ Faculty of Medicine / Zawia University Zawiya, Libya

${ }^{2}$ Faculty of Science /Zawia University Zawiya, Libya

${ }^{3}$ Medical research center / Zawia city Zawiya, Libya

${ }^{4}$ Faculty of Science /Gharyan University Zawiya, Libya

DOI: $10.36348 /$ SJMPS.2019.v05i10.002

| Received: 25.08.2019 | Accepted: 13.09.2019 | Published: 11.10.2019

*Corresponding author: Ahlam Rashed

\section{Abstract}

Many medicinal plants were studied for their phytochemical contents. Moringa oleifera is one of these plants. It is widely distributed in tropical and subtropical areas. It has an important medical uses with high nutritional value. This study, primarily aimed to carry out a preliminary phytochemical screening to detect the major classes of bioactive compounds presented in Moringa oleifera dry leaves. Many solvents (Methanol, ethanol, ethanol acetate and water) used to determine the best solvent can be used for extraction and to perform thin layer chromatography (TLC) profiling of all sequential extracts. TLC was carried out in silica gel plates using many mobile phase protocols, chloroform: methanol: ethanol (1:1:1), (2:2:0.5) and Chloroform: Glacial acetic acid: methanol (4:5:1) and use four mentioned solvents. Phytochemical screening of four extracts showed the presence of alkaloids, flavonoids, tannins, and phenols in all solvent extracts. However, the concentration of these compounds is more with ethanol and ethanol acetate extracts (+++). As well as thin layer chromatographic studies of the Moringa olifera leave extract (methanol, ethanol, ethanol acetate and water) constituted different colored phytochemical compounds with different Rf values. Methanol and ethyl acetate extracts were the best which show 4 bands with Rf less than 1 while with ethanol extract 5 bands with Rf less than 1 and with water extract no band appeared by using chloroform: methanol: ethanol (1:1:1). Furthermore with the other mobile phases detect fewer bands with different solvent extracts. The results obtained in the present study revealed that the concentration of the bioactive compounds of Moringa oleifera leaves extracts by using ethanol and ethyl acetate as a solvent is more than the other solvents. On the other hand, TLC showed 4 bands with better Rf by using methanol and ethyl acetate extract. Conclusion, the methanol and ethyl acetate extracts for Moringa oleifer leaves contain a higher content of bioactive compounds, which can be used for further researches on this plant.

Keywords: Moringa oleifera Thin Layer.

Copyright @ 2019: This is an open-access article distributed under the terms of the Creative Commons Attribution license which permits unrestricted use, distribution, and reproduction in any medium for non-commercial use (NonCommercial, or CC-BY-NC) provided the original author and source are credited.

\section{INTRODUCTION}

For thousands of years the nature is the best origin for the traditional agents [1]. About 20\% of known plants have been used in pharmaceutical drug discovery and study their effectiveness of the bioactive compounds on the health care system such as harmful chronic diseases and cancer [2]. Moringa is a medicinal plant, which grow in tropical area and used as human food, medicine and in oil production [3]. The Thin layer chromatography (TLC) method is an important analytical tool for the separation, identification and estimation of different classes of bioactive compounds
[4]. The leaves of Moriga Oleifera, in particular, have been found to contain phenolics and flavonoids [5]. These compounds have various biological properties, such as antioxidant, anticarcinogenic, immunomodulatory, antidiabetic, antiatherogenic, and hepatoprotective functions [6]. These phytoconstituents give special characteristics and properties to plants. Therefore, the analysis of these bioactive compuonds in plants would help in determining various biological activities of plants [7]. Although, the biological importance of Moringa Oliefera has been learned additional knowledge still secured. So, in recent years, 
more attention has been pointed towards identification of plants with antioxidant ability which could be used for human consumption [8]. These phytoconstituents confer specific characteristics and properties to plants. Although, much has been learned about the biological aspects of M. oleifera additional knowledge remains to be secured. Therefore, in recent years; considerable attention has been directed towards identification of plants with antioxidant ability that may be used for human consumption. Thus, the aims of present study are to investigate the phytochemical profile and thin layer chromatography (TLC) studies of M. oleifera leaves grown in Libya by using many types of solvents so as to know various constituents present in it and hence to evaluate the medicinal potential of the plant and justify its traditional use.

\section{MATERIALS AND METHODS \\ Procurement of Experimental Plant}

The experimental plant $M$. oleifera was collected from a farm at Tripoli Libya Moriga Oleifera leaves was healthy and uninfected. The leaves were air dried at room temperature and grinded in powder form [9]. $5 \mathrm{gm}$ of the powdered leaves were separately extract with 4 type of solvents $50 \mathrm{ml}$ each

\section{Sequential Extraction of M. oleifera leaves}

The dried M. oleifera leaves were powdered with the help of mixer grinder. The powdered leaves were then extracted by using sequential solvents that were pet methanol, ethanol, ethyl acetate and water for $24 \mathrm{~h}$. The extract was separately filtered using Whitman's no1 filter paper.

\section{Preliminary Phytochemical Screening of Successive}

\section{Extracts of M. oleifera Pods}

Qualitative phytochemical analysis of $\mathrm{M}$. oleifera leaves was carried out using standard procedures to identify the constituents, Alkaloids, Flavonoids, Tannin and Phenol as described by [10].

\section{Test for alkaloids}

$1 \%$ of HCL prepared and added to the Moringa Oleifera extract in test tube and heat it for $20 \mathrm{~min}$ with gently shake then leave it to cool a bit. Take $1 \mathrm{ml}$ of the extract and added few drops of Wagner's reagent notice a creamy brown indicate the presence of Alkaloids.

\section{Test for flavonoids}

$3 \mathrm{ml}$ of Moringa Oleifera extract added to $10 \mathrm{ml}$ of distilled water and mix it well notice a yellow color indicate the presence of Flavonoid.

\section{Test for tannins}

$2 \mathrm{ml}$ of Moringa Oleifera extract and put it in test tube and gently heat it for 2 min .added 3 drops of Ferric chloride notice orange color indicate the presence of Tannin.

\section{Test for phenols}

$3 \mathrm{ml}$ of Moringa Oleifera extract added to $5 \mathrm{ml}$ distilled water added few drops of $5 \%$ Ferric chloride notice dark green indicate the presence of phenol.

\section{Chromatographic purification}

TLC was carried out to isolate the principle components that were presents in most effective extracts of plants the TLC was used by different solvent systems

\section{Solvent phase}

Chloroform: methanol :ethanol ( 1:1:1 ), Chloroform: methanol: ethanol( 2:2:0.5) Chloroform : Glacial acetic acid :methanol ( 4:5:1) [11].

\section{Methods}

The plant extract were with 4 different solvent each extract applied on - pre coated TLC plate by using capillary tubes. Drawing a light line on the plate and dots to know the place of each extract applied on the plate. After using each mobile phase the TLC plate were air dried and observed under ultra violet light. They were later sprayed with iodine vapous for the development of the separated bands the movement was expressed by its retention factor (Rf ) values were calculated for different sample.

\section{$\mathbf{R f}=\frac{\text { Distance traveled by the solute }}{\text { Distance traveled by the solute front TLC plate }}$}

\section{Analyte detection}

After drying the plate and exposed with iodine vapous all plates were visualized with the help of UV and all different spots that were observed was calculated.

\section{RESULT}

\section{Phytochemical Screening of Sequential Extracts of} M. oleifera leaves

Phytochemical screening of the sequential extract of M. oleifera leaves showed the presence of various bioactive components of which phenol, alkaloids, flavonoids and tannin are the most prominent 
components and the result of phytochemical test is presented in Table 1. Among these phytochemical tests, ethanol and ethyl acetate extract were found to contain maximum of alkaloids, flavonoids, tannin and phenol in comparison with other solvents. All these phytochemicals possess good antioxidant activities and has been reported to exhibit multiple biological effects including anti-inflammatory and antitumor activities.

Table-1: Qualitative phytochemical screening of sequential extracts of Moringa oleifera leaves

\begin{tabular}{|l|c|c|c|c|}
\hline Solvent used & Alkaloids & Flavonoids & Tannin & Phenol \\
\hline Methanol & ++ & +++ & ++ & +++ \\
\hline Ethanol & +++ & +++ & +++ & +++ \\
\hline Ethyl acetate & +++ & +++ & +++ & +++ \\
\hline Water & ++ & +++ & +++ & +++ \\
\hline
\end{tabular}

\section{Chromatographic Purification: TLC}

Chloroform: Methanol: Ethanol (1:1:1) TLC of pet methanol extract of $\mathrm{M}$. oleifera leaves revealed the presence of 4 compounds having $\mathrm{Rf}$ values of 0.01 , $0.89,0.92$ and 0.93 respectively when a solvent phase of was used. With ethanol extract by same solvent showed 5 bands having Rf values of $0.046,0.66,0.83$, 0.900 .93 respectively. With ethyl acetate extract shows 4 bands having Rf of 0.046, 0.50, 0.56, 0.89 . However, with water extract shows no bands.

Chloroform: methanol: ethanol (2:2:0.5) Methanol extract shows 3 bands having Rf values of 0.090, 0.090, 0.81. With ethanol extract shows 3 bands having $\mathrm{Rf}$ values of $0.090,0.8,0.81$. With ethyl acetate shows no bands with water extract show 2 bands having Rf values of $0.63,0.76$

Chloroform: Glacial acetic acid: methanol (4:5:1) with methanol extract shows 2 bands having Rf values of $0.45,0.78$. while, Ethanol extract show no bands and with ethyl acetate extract shows 3 bands having $\mathrm{Rf}$ values of $0.4,0.76,0.83$. However with water extract show no bands

\section{DISCUSSION}

By using the qualitative phytochemical screening of plant extracts the chemical constituents can be provided for pharmacological and pathological discovery of the pharmaceutical agents [11]. In the present study, qualitative tests for all four extracts showed significant indication about the presence of metabolites. Alkaloids, phenols, tannins and Flavonoids were found to be present in the all the sequential extracts of $M$. oleifera leaves, with a maximum concentration by using ethanol and ethyl acetate solvents. TLC profiling of all 4 extracts gives an impressive result that directing towards the presence of number of phytochemicals. Various phytochemicals gives different $\mathrm{Rf}$ values in different solvent system. This variation in $\mathrm{Rf}$ values of the phytochemicals provides a very important clue in understanding of their polarity and also helps in selection of appropriate solvent system for separation of pure compounds by column chromatography. Mixture of solvents with variable polarity in different ratio can be used for separation of pure compound from plant extract. The selection of appropriate solvent system for a particular plant extracts can only be achieved by analyzing the Rf values of compounds in different solvent system $[8$, 12]. Different Rf values of the compound also reflect an idea about their polariy. This result will help in selection of appropriate solvent system for further separation of compound from these plant extracts.

\section{CONCLUSION}

The results revealed that $\mathrm{M}$. oleifera leaves as a rich source of bioactive compounds. These findings suggested that $M$. oleifera leaves have a potential source of natural antioxidant which have great importance as therapeutic agent for many chronic diseases. In addition, the methanol and ethyl acetate extracts for Moringa oleifera leaves contain a higher content of bioactive compounds, which can be used for further researches on this plant.

\section{REFRENCES}

1. Cragg, G. M., \& Newman, D. J. (2001). Natural product drug discovery in the next millennium. Pharmaceutical biology, 39(sup1), 817.

2. Naczk, M., Shahidi, F.(2006). Phenolics in cereals, fruits and vegetables: Occurrence, extraction and analysis.J. Pharm. Biomed. Anal, 41, 1523-1542.

3. Anwar, F., Latif, S., Ashraf, M., \& Gilani, A. H. (2007). Moringa oleifera: a food plant with multiple medicinal uses. Phytotherapy Research: An International Journal Devoted to Pharmacological and Toxicological Evaluation of Natural Product Derivatives, 21(1), 17-25.

4. Satyanarayana, V., \& Kumari, S. J. (2016). Preliminary phytochemical screening and TLC profile of selected four plants of Tirupati hills in Chitoor district, Andhra Pradesh. Journal of Pharmacognosy and Phytochemistry, 5(2), 259.

5. Mbikay, M. (2012). Therapeutic potential of Moringa oleifera leaves in chronic hyperglycemia and dyslipidemia: a review. Frontiers in pharmacology, 3, 24.

6. Khalafalla, M. M., Abdellatef, E., Dafalla, H. M., Nassrallah, A. A., Aboul-Enein, K. M., Lightfoot, D. A., ... \& El-Shemy, H. A. (2010). Active principle from Moringa oleifera Lam leaves effective against two leukemias and a 
hepatocarcinoma. African Journal of

Biotechnology, 9(49), 8467-8471.

7. Rajula, E., \& Ujwala, J. (2010). Phytochemical screening of Moringa oleifera using high performance thin layer chromatography. Plant Archives, 10(2), 749-751.

8. Sharma, V., Paliwal, R., Sharma, P., \& Sharma, S. (2011). Phytochemical analysis and evaluation of antioxidant activities of hydro-ethanolic extracts of Moringa oleifera Lam. pods. J Pharm Res, 4(2), 554-7.

9. Boots, A. W., Haenen, G. R., \& Bast, A. (2008). Health effects of quercetin: from antioxidant to nutraceutical. European journal of pharmacology, 585(2-3), 325-337.

10. Patel, K., Patil, A., Mehta, M., Gota, V., \& Vavia, P. (2014). Oral delivery of paclitaxel nanocrystal
(PNC) with a dual Pgp-CYP3A4 inhibitor: preparation, characterization and antitumor activity. International journal of pharmaceutics, 472(1-2), 214-223.

11. Paliwal, R., Sharma, V., \& Sharma, S. (2011). Elucidation of free radical scavenging and antioxidant activity of aqueous and hydro-ethanolic extracts of Moringa oleifera pods. Research Journal of Pharmacy and Technology, 4(4), 566571.

12. Suffredini, I. B., Sader, H. S., Gonçalves, A. G., Reis, A. O., Gales, A. C., Varella, A. D., \& Younes, R. N. (2004). Screening of antibacterial extracts from plants native to the Brazilian Amazon Rain Forest and Atlantic Forest. Brazilian journal of medical and biological research,37(3), 379384. 